\title{
Latest Results in SLAC 75 MW PPM Klystrons
}

\author{
D. Sprehn, G. Caryotakis, A. Haase, E. Jongewaard, L. Laurent, C. \\ Pearson, R. Phillips \\ Stanford Linear Accelerator Center \\ Stanford University, Stanford, CA 94309
}

\begin{abstract}
75 MW X-band klystrons utilizing Periodic Permanent Magnet (PPM) focusing have been undergoing design, fabrication and testing at the Stanford Linear Accelerator Center (SLAC) for almost nine years. The klystron development has been geared toward realizing the necessary components for the construction of the Next Linear Collider (NLC). The PPM devices built to date which fit this class of operation consist of a variety of $50 \mathrm{MW}$ and $75 \mathrm{MW}$ devices constructed by SLAC, KEK (Tsukuba, Japan ) and industry. All these tubes follow from the successful SLAC design of a 50 MW PPM klystron in 1996. In 2004 the latest two klystrons were constructed and tested with preliminary results reported at EPAC2004. The first of these two devices was tested to the full NLC specifications of $75 \mathrm{MW}, 1.6$ microseconds pulse length, and $120 \mathrm{~Hz}$. This $14.4 \mathrm{~kW}$ average power operation came with a tube efficiency $>50 \%$. The most recent testing of these last two devices will be presented here. Design and manufacturing issues of the latest klystron, due to be tested by the Fall of 2005, are also discussed.
\end{abstract}

Keywords: PPM, Klystron, High Power Microwave, NLC.

PACS: 84.40.Fe, 84.47.+w.

\section{INTRODUCTION - A SOURCE FOR THE NEXT LINEAR COLLIDER}

The Next Linear Collider (NLC), as envisioned by the physics community in the United States up until late 2004, initially required several thousand high power X-band klystrons operating at approximately $50 \mathrm{MW}$ with at least a $1.2 \mu \mathrm{s}$ pulsewidth. In order to upgrade to $1 \mathrm{TeV}$ energy levels, the NLC baseline design required several thousand 75 MW klystrons. Because of the prohibitive cost of operating solenoids and the complexities with superconducting magnet systems, an investigation into the merits of PPM focusing was begun. The major advantage with PPM focusing is in the cost savings of DC power, potentially tens of millions of dollars per year. However, stability and beam transport issues favor a solenoid over a PPM design.

The reasons the $75 \mathrm{MW}$ power level was chosen, rather than some other level, are well-known to klystron designers. There are tradeoffs between perveance, efficiency and power with practical choices of beam voltage, magnetic field and current density. The choice of drift tube size leads to reasonable gun convergence ratios, current and power densities, coupling coefficients, as well as the limitations of any PPM structure. Finally, gradients in the penultimate and output, for SLAC-type pseudo traveling-wave structures, are pushed to the limit at least as far as current convention finds acceptable. Thus at this frequency and pulse-length a $75 \mathrm{MW}$ source appeared to 
be at the limit for a conventional linear round-beam fundamental-mode device with well-behaved gain, efficiency and bandwidth performance.

\section{Brief History of NLC Sources}

After an initial call for a "4x SLAC" device (4 X $2856 \mathrm{MHz}$ ) some effort went into the investigation of sheet-beam and cross-field [1] devices. Shortly thereafter, a $100 \mathrm{MW}$-level solenoid focused klystron development program [2] ensued with various levels of success utilizing a number of extended-interaction output coupling schemes. Due to a realization of the trade-offs discussed previously and data from the $100 \mathrm{MW}$ effort, the design focus was set to $50 \mathrm{MW}$. The fourth of these $50 \mathrm{MW}$ designs [3], the XL4, has been the rf source of choice for X-band component and accelerator development for the last 7 years. At approximately the same time the XL4 was undergoing testing, the first 50 MW PPM klystron [4] was designed and tested. This first PPM klystron, the 50XP, met and exceeded the target specifications and operated at $50 \mathrm{MW}$ at $2.82 \mu$ s for approximately 1000 hours. Subsequent autopsy of the device showed relatively normal alterations to the circuit topology leading to the conclusion that a $50 \mathrm{MW}$ device was a viable option for the NLC. Two similar $50 \mathrm{MW}$ devices were built by industry and delivered to the SLAC Klystron Test Laboratory but unfortunately suffered from manufacturing related vacumn problems.

For approximately the last 6 years, until the program was essentially halted in late 2004 with the end of the NLC program, two diodes and seven klystrons aimed at a $75 \mathrm{MW}$ target specification were constructed. Six of these devices were constructed in the last three years with the last two awaiting test since October 2004.

\section{XP PPM KLYSTRONS}

TABLE 1. Basic XP3 parameters.

\begin{tabular}{lc}
\hline Parameter & Value \\
\hline Frequency & $11.424 \mathrm{GHz}$ \\
Beam voltage & $490 \mathrm{kV}$ \\
Beam Current & $257 \mathrm{~A}$ \\
RF Power at loads & $75 \mathrm{MW}$ \\
Pulse length & 1.6 to $3.2 \mu \mathrm{s}$ \\
PRF & $120 \mathrm{~Hz}$ \\
Average Power & 14 to $29 \mathrm{~kW}$ \\
Gain & $\sim 55 \mathrm{~dB}$ \\
Efficiency & $\sim 55 \%$ \\
Bandwidth & $120 \mathrm{MHz}$ \\
\hline
\end{tabular}

With allowances for a larger gun, modest alterations to the rf circuit and the higher magnetic field requirement, the 75XP designs closely resemble that of the 50XP design. In order to achieve the power specification it was necessary to increase the 0.6 microperveance gun of the $50 \mathrm{XP}$ to $0.75 \mu \mathrm{K}$ and raise the beam voltage from $465 \mathrm{kV}$ to $490 \mathrm{kV}$. All the 75XP devices used the same parameter set as shown in Table 1 with the exception of the pulse length, which depended upon the changing NLC machine design parameters. 
Construction of the first tube, the XP1, was delayed due to a problem with the quality of the ring-shaped magnets from the vendor. Errors in the magnetization alignment, thermal stability and uniformity of the material were unappreciated and overlooked such that three sets of these magnets were fabricated with none of them conforming to the requested specification. Though interception and thermal load were higher than the design called for, it can be seen in Figure 1 that the power output was more than adequate to achieve the tube specification. With this information in hand it was clear that the development of a more robust design, the XP3, could proceed.

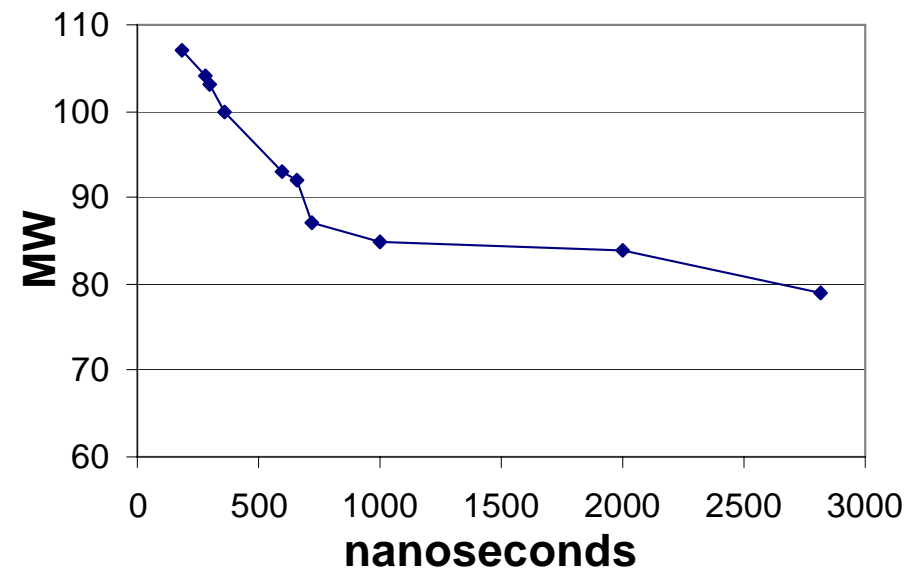

FIGURE 1. Peak power output vs. pulse length for the first 75 MW PPM klystron (XP1).

\section{XP3 Klystrons}

Several design changes were made to the XP3 design that distinguished it from its predecessor the XP1. Besides a simplified and more compact electron gun design to reduce the cost, the major differences were in the mechanical realization of the PPM structure and the shape of the magnetic field.

\section{Magnetic Structure Details}

Based on prior experience with the XP1 it was concluded that it is highly desirable to test the magnetic circuit before tube operation. In addition, it was realized that to be cost-competitive with solenoidal designs the PPM magnet stack should be reusable with a minimal amount of labor involvement. To accomplish this the structure took the form of clam-shell halves containing all the field-forming components that could be built and tested apart from the klystron vacuum envelope. Simulations and results from a test structure indicated that the transverse fields introduced by splitting the magnet structure could be controlled by precise fabrication and alignment. Thermal design of this clamp-on magnet structure, for any thermal dissipation along the klystron drift tube, also appeared under control. Part of such a test structure is shown in Figure 2 where an assembled "clam-shell" is shown on the right and a sample block containing one half of a small test unit is on the left. The actual implementation of the structure took various forms during the XP3 series of devices. 
Tests on PPM magnet stacks, representing a reasonable approximation of how the stacks were actually applied to the devices, were not a simple matter. Solenoidal designs generally call for transverse to axial field ratios on order of $0.2 \%$. The particle dynamics of a PPM device are not straightforward in that the transverse field from one magnet cell to the next has a magnetic vector that can vary in angle and strength, and there is also concentricity to consider. Historically such field errors were compensated for by manually shunting the field while the tube is in operation. Because of the high $\mathrm{x}$-ray flux this latter option was unavailable and only great care in the manufacture of the device could reduce field errors to acceptable levels. In simulation [5] various combinations of field errors, even those 10 times as high as those found in solenoidal tubes, would allow full beam transport if the errors were known and aligned in specific combinations. However, for errors on the order of $0.2 \%$ without compensation the beam transport could suffer. Discerning these fields in the 0.375" diameter drift tube required 16 measurements at each point with appropriate rotation of both the probe and the magnetic circuit. Disassembly and reassembly of the structure and realignment of the probe and guide scheme pointed to an uncertainty in the measurements almost to the same degree as the field errors which were the object of the measurements. In the final analysis a reasonable conclusion was that the field errors were somewhere between $0.2 \%$ and $1.2 \%$ as installed on the XP3-1, XP3-2 and XP3-3 klystrons.

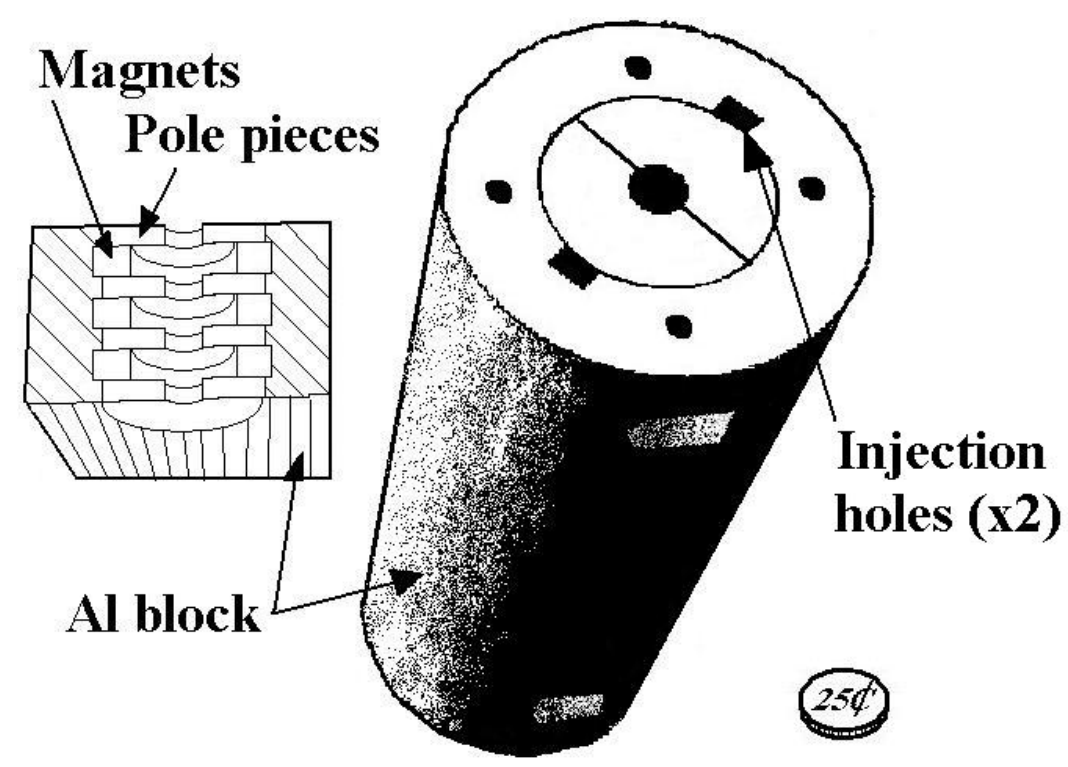

FIGURE 2. Magnet test structures for the XP3 design with magnets and pole pieces inserted into an aluminum block and epoxy applied.

\section{XP3 Testing and Comparison to Design}

During the manufacture of the first two of these tubes, known as the XP3-1 and XP3-2, the pulse length specification was doubled from $1.6 \mu$ s to $3.2 \mu$ s. Some outside vendor involvement had previously been initiated and CPI delivered an rf 
section and clam-shell structure which were subsequently made part of the XP3-2. The gun, as mentioned previously, had been reduced in size and optimized with little overhead in the engineered design such that the $3.2 \mu$ s target was a possible problem. Upon testing of the devices it was determined that XP3-1 had an oscillation in the output at $11.7 \mathrm{GHz}$ and XP3-2 had a gun oscillation at the end of the pulse which broke the gun ceramic. A third tube, XP3-3, was constructed which addressed both of these issues: an $11.7 \mathrm{GHz}$ loss cavity had been incorrectly installed on the XP3-1 and was correctly installed on XP3-3; and an additional gun loss ceramic was added to XP3-3 to address the gun instability. The XP3-3 was tested and operated at the full specification of $75 \mathrm{MW}$ load power at $120 \mathrm{~Hz}$ and $1.6 \mu \mathrm{s}$. It is of interest to note that between the XP3-2 and XP3-3 tests the NLC design was again changed back to $1.6 \mu \mathrm{s}$, which effectively returned the gun design to its original level of robustness.

Simulation using the PIC code MAGIC [6] has proven very successful for SLAC-built klystrons at frequencies from $476 \mathrm{MHz}$ to $11.424 \mathrm{GHz}$ in predicting tube power, bandwidth, thermal load and transmission. A simulation of the bunching and output section of the XP3-3, after completing a matrix of several hundred simulations to slightly vary the tuning of the rf circuit, is shown in Figure 3. This simulation predicted $76 \mathrm{MW}$ at $490 \mathrm{kV}$, which was well within the measurement accuracy in the lab.

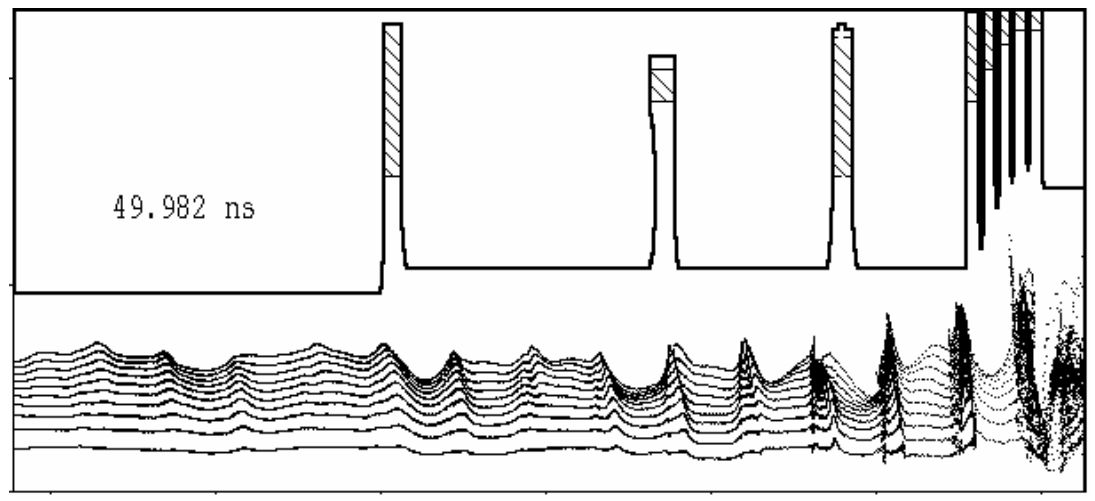

FIGURE 3. XP3-3 Simulation using 2.5D PIC code MAGIC after full convergence (50 ns) with 2D magnetic field input. Picture is expanded 12:1 in the transverse dimension to show beam detail.

Despite the success of XP3-3, detailed measurements of intercepted current and power during testing revealed beam transmission performance issues that were less than optimal. With the clam-shell structure, it was not possible to directly observe the heating of the magnets under operation and a thermal runaway condition damaged the magnet at the fifth cavity. The stiffness and straightness of the relatively weak drift tube was also a concern; each of the clam-shell drift tubes had at least a 0.010" bend. It was decided to abandon the clam-shell design in favor of the more conventional approach where complete pole plates are brazed onto the drift tube. This approach would allow for direct thermal measurements of magnets and pole pieces, better thermal conductivity and a stiffer drift tube. 


\section{XP3-4 and XP3-5}

Two klystrons were manufactured, the XP3-4 and XP3-5, with integral pole pieces and individual magnets. Essentially no changes were made to the rf design other than larger radii on the output coupler irises to eliminate activity found during autopsy on XP3-3. Due to time constraints the first of these, the XP3-4, was tested with forced air rather than water cooling but still operated at the full specification as shown in Figure 4, albeit with slightly less efficiency than previously observed since it was necessary to raise the beam voltage from $490 \mathrm{kV}$ to $506 \mathrm{kV}$. Saturated rf power versus beam voltage from $410 \mathrm{kV}$ to $510 \mathrm{kV}$ was measured and found to be extremely linear. Gain and power output, as in Figure 5, were found to be smooth and free of any indicators of competing modes within the circuit.

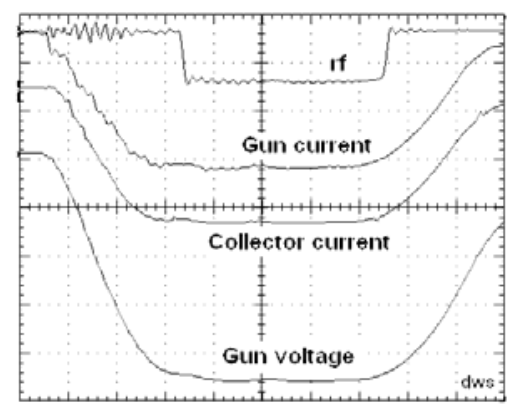

FIGURE 4. XP3-4 operation at $75 \mathrm{MW}, 506 \mathrm{kV}, 120 \mathrm{~Hz}$ and $1.62 \mu$ s with $\sim 60 \mathrm{~dB}$ of gain and $50 \%$ efficiency. Integrated time-averaged measurements indicate a $1.3 \%$ beam loss.

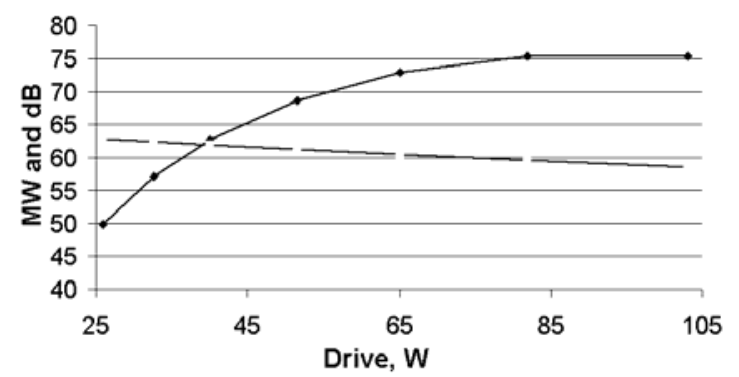

FIGURE 5. XP3-4 Output versus drive at $510 \mathrm{kV}, 120 \mathrm{~Hz}$ and $1.62 \mu \mathrm{s}$. Spurious signals were measured $>35 \mathrm{~dB}$ below the carrier from the gun, output waveguide and collector.

One of the most impressive aspects of this tube is the lack of beam current interception at full rf power. This is attributed to better control over the beam dynamics afforded by the integral pole-piece design as opposed to the clam-shell design. Measurements of the individual magnets on a test structure indicated that the transverse fields were from $0.1 \%$ to $0.6 \%$ along the drift tube. This represents the lowest ratios seen in the 75XP devices. The $1.3 \%$ beam interception at saturation is considered, even for low power PPM devices, to be quite small. In contrast, the 50XP lost $\sim 1 \%$ of the total beam power and the beam current lost was $\sim 7 \%$. The XP3-4 power interception was unmeasurable within the accuracy of the diagnostics but was likely much less than $1.3 \%$ since it is the slower electrons that are generally 
intercepted. However the range of available gun coil settings for such low interception values was much smaller than expected, and it is believed that beam scalloping and field errors were still issues that could be improved upon.

The latest klystron to be tested, the XP3-5, began testing [7] in the summer of 2004 and initially behaved similarly to the XP3-4. Both klystrons had excellent rf performance with $\sim 100 \mathrm{MHz}$ bandwidth, spurious signals below $-35 \mathrm{~dB}$, and approximately the same gain of $\sim 60 \mathrm{~dB}$. Nevertheless, after the pulse energy was increased during testing of the XP3-5 it was determined that there was breakdown somewhere between the output cavity and the rf loads. It was thought that this was at one of the windows and an x-ray scintillator array was installed to determine the exact location. Unfortunately, at the time of this paper the klystron is still awaiting test.

Though successful as the XP3-4 and XP3-5 were in terms of beam formation, transport and rf circuit design, rf breakdown appeared to be a limiting factor in tube longevity. Particularly during 24-hour $60 \mathrm{~Hz}$ operation of the XP3-4 the peak power on-time continually degraded over several weeks. This tube currently is awaiting autopsy to determine where in the output assembly this damage was occurring. Whether this breakdown was due primarily to the beam transport asymmetries or from a fundamental limitation of the materials and geometries used is unclear. In the past few years an intensive testing program of NLC accelerator sections and components, as well as renewed experimentation on the fundamental behavior of rf breakdown for high-power x-band components, has led the design team to believe that the chosen gradients for the 75XP designs lack sufficient engineering overhead for a production tube. Recent advances in output structure and coupler design could rectify this situation via a redesign of the power output extraction scheme. Such a redesign would have a goal of gradient reduction on order of $20 \%$.

\section{FUTURE WORK}

A significant alteration of the XP3 design was created in a new device known as the XP4. Changes included a further refinement of the magnetic structure details, provisions for thermal mapping of the drift tube, and changes to the gun to decrease the $\mathrm{Q}$ of any possible oscillating mode. Some alterations to the rf circuit were made to further reduce any beam expansion due to rf defocusing in the penultimate region. The XP4-1 klystron, principally constructed in 2004, is currently awaiting test. There are no other 75XP klystrons planned at SLAC due to the choice of $1.3 \mathrm{GHz}$ as the source for the $\mathrm{TeV}$ linear accelerator [8]. However, the klystron group at KEK in Tsukuba Japan has plans to continue this area of research [9] for at least the next two years.

\section{ACKNOWLEDGMENTS}

Over one hundred individuals have contributed in the design, fabrication, processing and testing of these devices. The majority of these individuals are still within the klystron department at SLAC. Special credit should go to Dr. George Caryotakis for his knowledge, perseverance and guidance in linear-beam technology, 
Robert M. Phillips for his championing of and knowledge of PPM technology, and Chris Pearson for his knowledge and management of the mechanical design and fabrication of SLAC klystrons. Of special note also are K. Eppley, R. Fowkes, S. Gold, E. Jongewaard, M. Harding, A. Haase, L. Laurent, T. Lee, D. Martin, J. Rominger, G. Scheitrum, R. Schumacher, Y. Shin, A. Vlieks, C. Wilsen, and E. Wright.

This work is supported by the Department of Energy under Contract DE-AC03$76 \mathrm{SF} 00515$.

\section{REFERENCES}

1. W.R. Fowkes et al., "RF Power Generation for Future Linear Colliders" Presented by W. Fowkes at BEAMS '90' 8th Int. Conf. on High Power Particle Beams, Novosibirsk, USSR, Jul 2-5, 1990. SLAC-PUB-5282.

2. G. Caryotakis et al., "Development of Multi-Megawatt Klystrons for Linear Colliders" Presented by G. Caryotakis at 1993 Particle Accelerator Conference (PAC 93), Washington, DC, 17-20 May 1993. Published in IEEE PAC 1993:1106-1108 (QCD183:P3:1993). SLAC-PUB-6168.

3. G. Caryotakis, "X Band Klystron Program at SLAC" Presented by G. Caryotakis at 3rd International Workshop on RF Pulsed Power Sources for Linear Colliders (RF 96), Hayama, Japan, 8-12 Apr 1996. SLAC-PUB-7146, Apr 1996. 9pp. In *Hayama 1996, Pulsed RF sources for linear colliders* 72-80.

4. D. Sprehn, G. Caryotakis, E. Jongewaard, R.M. Phillips, "Periodic Permanent Magnet Development for Linear Collider X Band Klystrons" Presented by D. Sprehn at 19th International Linear Accelerator Conference (Linac 98), Chicago, IL, 23-28 Aug 1998. Published in AIP Conf.Proc.474:31-40,1999 SLAC-PUB-7925, Sep 1998. 6pp.

5. Y. Shin (Seoul Natl. U.), G. Scheitrum, D. Sprehn (SLAC), "Modeling of Beam Transport in Transverse Magnet Fields" Presented by D. Sprehn at 2003 IEEE International Conference on Plasma Science, Jeju, South Korea, 2-5 Jun 2003. SLAC-REPRINT-2003-087, Jun 2003.

6. B. Goplen, L. Ludeking, D. Smithe, and G. Warren, "User-Configurable MAGIC Code for Electromagnetic PIC Calculations,” Computer Physics Communications, Vol. 87, Nos. 1 \& 2, May 1995, pp. 54-86.

7. D. Sprehn et al., "Current Status of the Next Linear Collider X-Band Klystron Development Program" Presented by D. Sprehn at the 9th European Particle Accelerator Conference (EPAC 2004), Lucerne, Switzerland, 5-9 Jul 2004, EPAC-2004-TUPKF063, Jul 2004. 3pp. SLAC-PUB11162.

8 ICFA, "International Panel Recommends Cold Technology for Future Particle Accelerator," Press Release in the Interaction Point. Interactions News Wire \#51-04, 20 August 2004. http://www.interactions.org

9. S. Matsumoto et al., "Development of PPM-focused X-band Pulse Klystron," Particle-AcceleratorSociety, Linac-Gijutsu-Kenkyukai-2005, pp.269-271, Tosu, Japan, July 20 - 22, 2005. 